Pacific Journal of Mathematics

DENDRITES, DIMENSION, AND THE INVERSE ARC 


\title{
DENDRITES, DIMENSION, AND THE INVERSE ARC FUNCTION
}

\author{
JoHN JoBE
}

In this paper, the concept of an inverse arc function is introduced. An inverse arc function $f$ is a function such that for each arc $L$ in the range of $f$, there exists an arc $L_{1}$ in the domain of $f$ such that $f\left(L_{1}\right)=L$. It is proved that a dendrite $D$ is the continuous image of an inverse arc function $f$ with domain an arc $L$ if and only if $D$ has only a finite number of endpoints. Other results are obtained telling what dendrites can be ranges of continuous inverse arc functions having dendrites as domains.

The dimension raising ability of a continuous inverse arc function whose domain is a dendrite is questioned. It is proved that if $D$ is a dendrite with only a countable number of endpoints, then there does not exist a continuous inverse arc function $f$ with domain $D$ such that $\operatorname{dim} f(D) \geqq 2$. If a dendrite $D$ has uncountably many endpoints, then the question is left unanswered.

Basic theorems and definitions used are as stated in [3], [4], [5], and [6]. In particular, a continuum $M$ is a dendrite provided it is locally connected and contains no simple closed curve. A continuum is a compact closed connected set. Other characterizations of a dendrite are also used. Topological spaces considered are all separable metric spaces. If $x$ and $y$ are distinct points, then $x y$ will denote an arc with end points $x$ and $y$.

Definition. Let $f: X \rightarrow Y$ be a function from $X$ onto $Y$. Then, $f$ is an inverse arc function if and only if for each arc $L \subset Y$ there exists an are $L_{1} \subset X$ such that $f\left(L_{1}\right)=L$.

In this paper the class $\mathscr{D}$ of all dendrites is partitioned into two subclasses, $\mathscr{Y}$ and $\mathscr{C}$, such that

$$
\begin{aligned}
\mathscr{C}= & \{X: X \in \mathscr{D} \text { and there exists a continuous inverse are } \\
& \text { function, } f, \text { with domain an arc } A \text { and } f(A)=X\}
\end{aligned}
$$

and

$$
\mathscr{K}=\mathscr{D}-\mathscr{H} \text {. }
$$

Then, it is shown that

$$
\mathscr{H}=\{X: X \in \mathscr{D} \text { and } X \text { has only a finite number of endpoints }\} .
$$

and 
$\mathscr{K}=\{X: X \in \mathscr{D}$ and $X$ has infinitely many endpoints $\}$.

Further related results are found by studying the question: "Can each member $X \in \mathscr{D}$ be the domain of a continuous inverse arc function, $f$, with $\operatorname{dim} f(X) \geqq 2$ "? It is shown that each member, $X$, of the class of all dendrites with only countably many endpoints cannot be the domain of a continuous inverse arc function, $f$, with $\operatorname{dim} f(X) \geqq 2$. Thus, there remains open an interesting question for further study. That is, if $D$ is a dendrite with uncountably many endpoints, then does there exist a continuous inverse arc function, $f$, with domain $D$ such that $\operatorname{dim} f(D) \geqq 2$ ? This function, if it exists, would necessarily be a dimension raising function since the $\operatorname{dim} D=1$.

The following examples point out that there are continuous inverse arc functions that do raise dimension.

ExAmple 1. Let $n$ be any natural number. Professor Bing [1] has shown that there are $n$-dimensional hereditarily indecomposable continua in $E_{n+1}$. Let $M_{n}$ be such a hereditarily indecomposable continuum in $E_{n+1}$. Let $P_{n}$ be a locally connected continuum in $E_{n+1}$ such that $M_{n} \subset P_{n}$. By the Hahn-Mazurkiewicz theorem there exists a continuous function, $h$, with domain the unit interval $I$ such that $h(I)=P_{n}$. Let $f$ be the restriction of $h$ to $h^{-1}\left(M_{n}\right)$. The domain of $f$ has dimension less than or equal to 1 since $\operatorname{dim} I=1$ and the dimension of $M_{n}$ (the range of $f$ ) is $n$. Thus, $f$ is a dimension raising continuous function when $n \geqq 2$. Since $M_{n}$ contains on arcs, then vacuously $f$ is a continuous inverse arc function that does raise dimension when $n \geqq 2$.

EXAMPLE 2. Let $S$ be any $n$-dimensional space such that each point of $S$ is contained in some are in $S$. Let

$$
\mathscr{Z}=\{L: L \subset S \text { and } L \text { is an arc }\} .
$$

Let

$$
T=\{(x, L): L \in \mathscr{A}, x \in L\}
$$

and $P: T \rightarrow S$ be the projection function such that $P(x, L)=x . \quad$ A basis, $\sigma$, for a topology for $T$ can be defined using $P$. Let

$$
\begin{aligned}
\sigma= & \left\{A_{L}: L \in \mathscr{A}, A_{L} \subset T \cap(S \times\{L\}), P\left(A_{L}\right) \cap L\right. \\
& \text { is an interval on } L \text { that is open relative to } L\}
\end{aligned}
$$

and $\tau$ bo the topology for $T$ generated by $\sigma$. The function $P: T \rightarrow S$ is a continuous inverse arc function from $T$ onto $S$ where the topology for $T$ is $\tau$. By observing that the boundary of each member of $\sigma$ has 
at most two points, it then follows that $\operatorname{dim} T=1 . \quad$ Since $\operatorname{dim} T=1$, then for any natural number $n \geqq 2, P$ is a dimension raising continuous inverse arc function.

Example 2 points out that given any $n$-dimensional space $S$ such that each point of $S$ is contained in some arc in $S$, then there exists

(1) a 1-dimensional space $T$ and

(2) a continuous inverse arc function, $P: T \rightarrow S$, onto $S$.

2. Inverse arc functions onto dendrites. A simple property about inverse arc functions is stated first as Theorem 1.

THEOREM 1. If $f: X \rightarrow Y$ and $g: Y \rightarrow Z$ are two inverse arc functions, then $g f: X \rightarrow Z$ is an inverse arc function.

Lemma 1. Let $D$ be a dendrite, $p \in D$, and $K$ the set of endpoints of $D$. Then

$$
D=\bigcup_{x \in K} p x
$$

Theorem 2 says that a dendrite with only a finite number of endpoints is a member of $\mathscr{H}$.

THEOREM 2. If $D$ is a dendrite with only a finite number of endpoints, $z \in D$, and $A=a b$ an arc, then there exists a continuous inverse arc function, $f$, with domain $A$, range $D$, and

$$
f(a)=f(b)=z \text {. }
$$

Proof. Without loss of generality consider the arc $A$ as the unit interval $I=[0,1]$. Suppose that $D$ has $n$ endpoints and denote these endpoints as $K=\left\{x_{1}, \cdots, x_{n}\right\}$. Lemma 1 implies that

$$
D=\bigcup_{i=2}^{n} x_{1} x_{i}
$$

This proof can be done by induction. If $n=2$ and we assume that $z \notin K$, then partition $I$ with the partition $P=\left\{a_{0}, a_{1}, a_{2}, a_{3}\right\}$ where $a_{0}=0, a_{1}=1 / 3, a_{2}=2 / 3$, and $a_{3}=1$.

Define

(1) $f_{1}:[0,1 / 3] \rightarrow D$ such that $f_{1}$ is a homeomorphism and $f_{1}(0)=$ $z, f_{1}(1 / 3)=x_{1}$, and $f_{1}([0,1 / 3])=z x_{1}$,

(2) $f_{2}:[1 / 3,2 / 3] \rightarrow D$ such that $f_{2}$ is a homeomorphism and $f_{2}(1 / 3)=$ $x_{1}, f_{2}(2 / 3)=x_{2}$, and $f_{2}([1 / 3,2 / 3])=x_{1} x_{2}$, and

(3) $f_{3}:[2 / 3,1] \rightarrow D$ such that $f_{3}$ is a homeomorphism and $f_{3}(2 / 3)=$ $x_{2}, f_{3}(1)=z$, and $f_{3}([2 / 3,1])=x_{2} z$. 
Now, let $f:[0,1] \rightarrow D$ be the function defined such that $f(x)=$ $f_{i}(x)$ for the appropriate natural number $i$. Clearly, $f$ is a continuous inverse arc function with domain $I$ such that $f(I)=D$ and $f(0)=$ $f(1)=z$.

If $z \in K$ then partition $[0,1]$ with $P=\left\{a_{0}, a_{1}, a_{2}\right\}$ where $a_{0}=0$, $a_{1}=1 / 2$, and $a_{2}=1$. Without loss of generality suppose $z=x_{1}$.

Define

(1) $f_{1}:[0,1 / 2] \rightarrow D$ such that $f_{1}$ is a homeomorphism and $f_{1}(0)=$ $x_{1}=z, f_{1}(1 / 2)=x_{2}$, and $f([0,1 / 2])=x_{1} x_{2}$, and

(2) $f_{2}:[1 / 2,1] \rightarrow D$ such that $f_{2}$ is a homeomorphism and $f_{2}(1 / 2)=$ $x_{2}, f_{2}(1)=x_{1}=z$, and $f_{2}([1 / 2,1])=x_{2} x_{1}$.

Now, let $f:[0,1] \rightarrow D$ be the function defined such that $f(x)=$ $f_{i}(x)$ for the appropriate natural number $i$. Clearly, $f$ is a continuous inverse arc function with domain $I$ such that $f(I)=D$ and $f(0)=$ $f(1)=z$.

Therefore, if $n=2$, then the theorem is true.

Suppose that the theorem is true for $n=k-1$. Now, let

$$
D=\bigcup_{i=2}^{k} x_{1} x_{i}
$$

Let $p$ be the first point on

$$
x_{k} x_{1} \cap\left(\bigcup_{i=2}^{k-1} x_{1} x_{i}\right)
$$

from $x_{k}$ to $x_{1}$. The induction hypothesis says that there exists

$$
g: I \longrightarrow \bigcup_{i=2}^{k-1} x_{1} x_{i}
$$

a continuous inverse arc function onto

$$
\bigcup_{i=2}^{k-1} x_{1} x_{i}
$$

such that $g(0)=g(1)=p$. Let $I_{1}=[0,2]$ and partition $[1,2]$ with the partition $P=\left\{a_{0}, a_{1}, \cdots, a_{2 k}\right\}$ where $a_{0}=1, a_{1}=2 k+1 / 2 k, \cdots$, $a_{j}=2 k+j / 2 k, \cdots, a_{2 k}=2$ and denote $\Delta_{j}=\left[a_{j-1}, a_{j}\right], j=1,2, \cdots, 2 k$.

Define

(1) $f_{1}: \Delta_{1} \rightarrow D$ such that $f_{1}$ is a homeomorphism, $f_{1}\left(a_{0}\right)=p$, $f_{1}\left(a_{1}\right)=x_{k}$, and $f_{1}\left(\Delta_{1}\right)=p x_{k}$,

(2) $f_{j}: \Delta_{j} \rightarrow D$ such that $f_{j}$ is a homeomorphism, $f_{j}\left(a_{j_{-1}}\right)=x_{k}$, $f_{j}\left(a_{j}\right)=x_{j / 2}$, and $f_{j}\left(\Delta_{j}\right)=x_{k} x_{j / 2}$ if $1<j<2 k$ and $j$ is even,

(3) $f_{j}: \Delta_{j} \rightarrow D$ such that $f_{j}$ is a homeomorphism, $f_{j}\left(a_{j-1}\right)=x_{j-1 / 2}$, $f_{j}\left(a_{j}\right)=x_{k}$, and $f_{j}\left(\Delta_{j}\right)=x_{j-1 / 2} x_{k}$ if $1<j<2 k$ and $j$ is odd, and

(4) $f_{2 k}: \Delta_{2 k} \rightarrow D$ such that $f_{2 k}$ is a homeomorphism, $f_{2 k}\left(a_{2 k-1}\right)=x_{k}$, $f_{2 k}\left(a_{2 k}\right)=p$, and $f_{2 k}\left(\Delta_{2 k}\right)=x_{k} p$. 
Let $h: I_{1} \rightarrow D$ be the function defined such that

$$
h(x)=g(x) \quad \text { if } x \in[0,1]
$$

or

$h(x)=f_{i}(x)$ for the appropriate natural number $i$ if $x \in[1,2]$.

Again, $h$ is a continuous function defined on the arc $I_{1}$ such that $h\left(I_{1}\right)=D$. Let $L$ be any $\operatorname{arc}$ in $D$. If

$$
L \subset \bigcup_{i=2}^{k-1} x_{1} x_{i}
$$

then the induction hypothesis implies that there exists an arc

$$
L_{1} \subset[0,1]
$$

such that $g\left(L_{1}\right)=L$. Since $h$ extends $g$ then $h\left(L_{1}\right)=L$. Otherwise, if $L$ is an arc in $D$ such that

$$
L \not \subset \bigcup_{i=2}^{k=1} x_{1} x_{i},
$$

then there exists a natural number $j$ such that $L \subset x_{j} x_{k}$. Now, note that $f_{2 j}\left(\Delta_{2 j}\right)=x_{k} x_{j}$ and since $f_{2 j}$ is a homeomorphism on $\Delta_{2 j}$ then there exists an arc $L_{1} \subset \Delta_{2 j}$ such that $f_{2 j}\left(L_{1}\right)=L$. Since $h$ extends $f_{2 j}$, then $h\left(L_{1}\right)=L$. Therefore, $h$ is an inverse arc function.

Let $z \in D$. If $z=p$ then $h$ is the desired function since $h$ is a continuous inverse arc function with domain an arc, range $D$, and $h(0)=h(2)=z$. Otherwise, suppose $z \neq p$ and $\varepsilon>0$. Consider the closed interval $[-\varepsilon, 2+\varepsilon]$.

Define

(1) $h_{1}:[-\varepsilon, 0] \rightarrow D$ such that $h_{1}$ is a homeomorphism onto $z p$, $h_{1}(-\varepsilon)=z$, and $h_{1}(0)=p$, and

(2) $h_{2}:[2,2+\varepsilon] \rightarrow D$ such that $h_{2}$ is a homeomorphism onto $p z$, $h_{2}(2)=p$, and $h_{2}(2+\varepsilon)=z$.

As before, let $s:[-\varepsilon, 2+\varepsilon] \rightarrow D$ be the function defined such that

$$
s(x)=h(x) \quad \text { if } x \in[0,2]
$$

or

$s(x)=h_{i}(x)$ for the appropriate natural number $i$ if

$$
x \in[-\varepsilon, 0] \cup[2,2+\varepsilon] \text {. }
$$

Again, $s$ is a continuous function defined on $[-\varepsilon, 2+\varepsilon]$ such that $s([-\varepsilon, 2+\varepsilon])=D$. Since $s$ extends $h$, then $s$ is also an inverse arc function. The function $s$ is defined such that 


$$
s(-\varepsilon)=s(2+\varepsilon)=z .
$$

Let $A$ be any arc denoted $A=a b$. Let $r: A \rightarrow[-\varepsilon, 2+\varepsilon]$ be a homeomorphism onto $[-\varepsilon, 2+\varepsilon]$. Now define $f$ to be the function $r s: A \rightarrow D$ and then $f$ is the function desired to prove this theorem by induction.

COROLlary 1. If $D$ is a dendrite with only a number of endpoints, $A=a b$ an arc, and $z_{1}, z_{2} \in D$, then there exists a continuous inverse arc function, $f$, with domain $A$, range $D, f(a)=z_{1}$, and $f(b)=z_{2}$.

Proof. This corollary can be proved in a similar way to that used to prove Theorem 2.

Lemma 2 can be easily proved and is an aid in the proof of Theorem 3.

Lemma 2. If $f: X \rightarrow Y$ is a continuous inverse arc function such

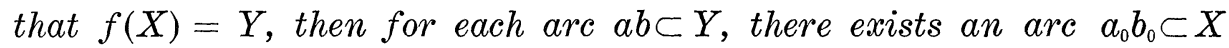
such that

(1) $f\left(a_{0} b_{0}\right)=a b$,

(2) $f\left(a_{0}\right)=a, f\left(b_{0}\right)=b$, and

(3) if $x \in a_{0} b_{0}, x \neq a_{0}$, and $x \neq b_{0}$, then $f(x) \neq a$ and $f(x) \neq b$.

THEOREM 3. Let $D$ be a dendrite with infinitely many endpoints and $I$ the unit interval. Then there does not exist $f: I \rightarrow D$ a continuous inverse arc function such that $f(I)=D$.

Proof. Let $y$ be a cut point of $D$ and $D-y=A \cup B$ sep. Without loss of generality suppose that $B$ contains infinitely many endpoints of $D$. Pick a point $z \in A$ and a countable infinite subset of endpoints contained in $B$ and name this subset, $\left\{y_{n}\right\}$. Now suppose that there exists $f: I \rightarrow D$ a continuous inverse arc function such that $f(I)=D$. Define

$$
K=\bigcup_{n=1}^{\infty} z y_{n}
$$

Using Lemma 2 we can obtain for each $n$ an arc $p_{n} x_{n} \subset I$ such that $f\left(p_{n} x_{n}\right)=z y_{n}$ having the properties stated in Lemma 2. Let

$$
\mathscr{A}_{1}=\left\{p_{n} x_{n}: n=1,2, \cdots\right\}
$$

and define $\mathscr{A}_{2} \subset \mathscr{A}_{1}$ such that $p_{1} x_{1} \in \mathscr{A}_{2}$. Suppose that for some $n$ it has been decided for each $k<n$ whether or not $p_{k} x_{k} \in \mathscr{A}_{2}$. Then $p_{n} x_{n} \in \mathscr{A}_{2}$ if and only if $p_{n} x_{n} \cap p_{k} x_{k}=\varnothing$ for each $k<n$ such that 
$p_{k} x_{k} \in \mathscr{A}_{2}$. By induction $\mathscr{A}_{2}$ is defined.

We show that no more than one member of $\mathscr{A}_{1}$ intersects another member of $\mathscr{A}_{1}$. Suppose that $p_{i} x_{i}$ and $p_{j} x_{j}$ are distinct members of $\mathscr{A}_{1}$ such that $p_{i} x_{i} \cap p_{j} x_{j} \neq \varnothing$. Since for each $k$, no point of

$$
p_{k} x_{k}-\left\{p_{k}, x_{k}\right\}
$$

maps onto either $z$ or $y_{y}$, then the only way that $p_{i} x_{i} \cap p_{j} x_{j} \neq \varnothing$ is for $p_{i}=p_{j}$ and without loss of generality $x_{i}<p_{i}<x_{j}$. (Note that if $p_{i}=p_{j}<x_{i}<x_{j}$ or if $x_{j}<x_{i}<p_{i}=p_{j}$ we we will reach a contradiction by again arguing as is in this paragraph below.) That is, the $\operatorname{arcs} p_{i} x_{i}$ and $p_{j} x_{j}$ are the the closed intervals $\left[x_{i}, p_{i}\right]$ and $\left[p_{j}, x_{j}\right]$ respectively with $p_{i}=p_{j}$. The case is, that no other $p_{k} x_{k} \in \mathscr{A}_{1}$ is such that $p_{k} x_{k} \cap p_{i} x_{i} \neq \varnothing$, for if we assume so, then without loss of generality $p_{k} x_{k}=\left[x_{k}, p_{k}\right]$ and $p_{i} x_{i}=\left[x_{i}, p_{i}\right]$ is true which implies either $\left[x_{i}, p_{i}\right] \subset\left[x_{k}, p_{k}\right]$ or $\left[x_{k}, p_{k}\right] \subset\left[x_{i}, p_{i}\right]$. If $\left[x_{i}, p_{i}\right] \subset\left[x_{k}, p_{k}\right]$, then $f\left(x_{i} p_{i}\right)=z y_{i} \subset f\left(x_{k} p_{k}\right)=z y_{k}$. This says that $y_{i}$ is contained in an arc of $D$ and is not an and point of that arc. This is a contradiction to the definition of $y_{i}$ being an endpoint of $D$. Similarly, a contradiction can be reached if we assume that $\left[x_{k}, p_{k}\right] \subset\left[x_{i}, p_{i}\right]$. Thus, no more than one member of $\mathscr{A}_{1}$ intersects another member of $\mathscr{A}_{1}$.

Suppose that $\mathscr{A}_{2}$ is finite. The method of definition of $\mathscr{A}_{2}$ implies that there exists $N$ such that for each $n>N$,

$$
p_{n} x_{n} \in \mathscr{A}_{1}-\mathscr{A}_{2} \text {. }
$$

For convenience, denote $\mathscr{A}_{2}=\left\{p_{1} x_{1}, \cdots, p_{k} x_{k}\right\}$. Since

$$
P_{N+1} x_{N+1} \in \mathscr{A} 1-\mathscr{A}_{2},
$$

then there exists only one member of $\mathscr{A}_{2}$ intersecting $p_{N+1} x_{N+1}$ If there were more than one, then this would contradict the argument in the preceding paragraph. Likewise, $p_{N+2} x_{N+2}$ intersects only one member of $\mathscr{A}_{2}$. The member of $\mathscr{A}_{2}$ that $p_{N+2} x_{N+2}$ intersects is distinct from the one that $p_{N+1} x_{N+1}$ intersects for if not, then again the argument in the preceding paragraph would be contradicted. After $k$ considerations, the set of $\operatorname{arcs} L=\left\{p_{N+1} x_{N+1}, \cdots, p_{N+k} x_{N+k}\right\}$, has the property that each member of $L$ intersects one and only one distinct member of $\mathscr{L}_{2}$. This exhausts $\mathscr{A}_{2}$ and implies that $p_{N+k+1} x_{N+k+1}$ intersects no member of $\mathscr{A}_{2}$. This contradicts the definition of $\mathscr{A}_{2}$ and therefore $\mathscr{A}_{2}$ is infinite.

Since we know that $\mathscr{A}_{2}$ is an infinite set of mutually exclusive $\operatorname{arcs}$ in $I$, then we can pick a null sequenc $\mathscr{A} \subset \mathscr{A}_{2}$. Denote $\mathscr{A}$ by $\mathscr{A}=\left\{p_{n_{i}} x_{n_{i}}\right\}$ and let $z_{0}$ be a member of the limit set of $\mathscr{A}$. Assume that $f\left(z_{0}\right) \neq z$. Let $U$ be any open set such that $f\left(z_{0}\right) \in U$ and $z \notin U$. Since $f$ is continuous, then there exists an open set $V$ such that 
$z_{0} \in V$ and $f(V) \subset U$. Because $\mathscr{A}$ is a null sequence and $z_{0}$ is in the limit set of $\mathscr{A}$, then there exists an $i$ such that $p_{n_{i}} x_{n_{i}} \subset V$. Thus, $z=f\left(p_{n_{i}}\right) \subset f(V) \subset U$ which contradicts the definition of $U$. Thus, $f\left(z_{0}\right)=z$.

The set $A$ is an open set containing $z$ and since $f\left(z_{0}\right)=z$ there exists an open set $V$ such that $z_{0} \in V$ and $f(V) \subset A$. Again, because $\mathscr{A}$ is a null sequence and $z_{0}$ is in the limit set of $\mathscr{A}$ there exists an $i$ such that $p_{n_{i}} x_{n_{i}} \subset V$. Therefore,

$$
y_{n_{i}}=f\left(x_{n_{i}}\right) \subset f(V) \subset A
$$

which contradicts $y_{n_{i}} \in B$. This final contradiction completes the proof of the theorem.

Theorem 4. The subclasses $\mathscr{X}$ and $\mathscr{K}$ of $\mathscr{D}$ are characterized as

$$
\mathscr{H}=\{X: X \in \mathscr{D} \text { and } X \text { has only a finite number of endpoints }\}
$$

and

$$
\mathscr{K}=\{X: X \in \mathscr{D} \text { and } X \text { has infinitely many endpoints }\} \text {. }
$$

Proof. The proof of theorem is a consequence of Theorems 2 and 3.

Theorem 4 classifies dendrites into the class of all dendrites that are the range of an inverse arc function whose domain is an arc and the class of all those dendrites that cannot be the range of an inverse arc function with domain an arc. The remaining theorems in this section tell us what dendrites can be ranges of continuous inverse arc functions having dendrites as domains. In addition, Theorem 5 will be used as a tool to prove Theorem 8 in $\S 3$.

Lemma 3. If $D$ is a dendrite and $H$ is an uncountable collection of arcs, each contained in $D$, then there exists $z \in D$ such that $z$ is contained in uncountably many members of $H$.

THEOREM 5. Let $S$ be a topological space that contains a subspace $M$ where

$$
M=\bigcup_{y \in L} p y,
$$

$L$ is an uncountable subset of $S, p \in S$, and if $y_{1}$ and $y_{2}$ are distinct members of $L$, then $y_{1} \notin p y_{2}$ and $y_{2} \notin p y_{1}$. If $D$ is a dendrite with only countably many endpoints, then there does not exist $f: D \rightarrow S$, a continuous inverse arc function such that $f(D)=S$. 
Proof. If we assume the contrary, then for each arc $p y, y \in L$, there exists an arc $L_{y}=o_{p} x_{y} \subset D$ with the properties given by Lemma 2. That is, $f\left(L_{y}\right)=p y, f\left(o_{p}\right)=p$, and $f\left(x_{y}\right)=y$. Let $H=$ $\left\{L_{y}: y \in L\right\}$. Lemma 3 implies that there exists a point $z \in D$ such that $z$ is contained in uncountably many members of $H$. Let

$$
K=\left\{L_{y}: L_{y} \in H, z \in L_{y}\right\} \text {. }
$$

Note that $K$ is uncountable and that for each

$$
L_{y}=o_{p} x_{y} \in K, z x_{y} \subset L_{y} \text {. }
$$

If we denote the endpoints of $D$ as $\left\{d_{n}\right\}$, then Lemma 1 allows us to denote

$$
D=\bigcup_{n=1}^{\infty} z d_{n}
$$

Since $K$ is uncountable there will exist $L_{y_{1}}, L_{y_{2}} \in K$, a natural number $N$, and $y_{1} \neq y_{2}$ such that $x_{y_{1}}$ and $x_{y_{2}}$ are points on the arc $z d_{N}$. Without loss of generality suppose $x_{y_{1}}<x_{y_{2}}$ on $z d_{N}$ from $z$ to $d_{N}$. Thus, $z x_{y_{1}} \subset z x_{y_{2}}$ and

$$
f\left(z x_{y_{1}}\right) \subset f\left(z x_{y_{2}}\right) \subset f\left(L_{y_{2}}\right)=p y_{2} .
$$

This says that $f\left(x_{y_{1}}\right)=y_{1} \in p y_{2}$ which contradicts hypothesis of the theorem.

CoRollary 2. If $D$ is a dendrite with uncountably many endpoints and $D_{1}$ a dendrite with only countably many endpoints, then there does not exist $f: D_{1} \rightarrow D$, a continuous inverse arc function such that $f\left(D_{1}\right)=D$.

Proof. Pick a point $p \in D$. Denote $D$ by

$$
D=\bigcup_{y \in L} p y
$$

where $L$ is the set of endpoints of $D$. The hypothesis says that $L$ is uncountable. Theorem 5 then implies the desired result.

THEOREM 6. If $D$ is a dendrite with an infinite number of endpoints and $D_{1}$ is a dendrite with only a finite number of endpoints, then there does not exist $f: D_{1} \rightarrow D$, a continuous inverse arc function such that $f\left(D_{1}\right)=D$.

Proof. Assume that such a function $f$ does exist. Theorem 2 implies that there exists $g: A \rightarrow D_{1}$, a continuous inverse arc function such that $A$ is an arc and $g(A)=D_{1}$. Then $f g: A \rightarrow D$ is a con- 
tinuous inverse arc function such that $f g(A)=D_{\text {s }}$ which contradicts Theorem 3.

Lemma 4. If $D$ is a dendrite and $A$ is any arc contained in $D$, then $D$ is retractible to $A$.

THEOREM 7. If $S$ is a topological space that is retractible to an arc $L_{1}$ and $D$ is any dendrite with only a finite number of endpoints, then there exists $f: S \rightarrow D$, a continuous inverse arc function such that $f(S)=D$.

Proof. Since $S$ is retractible to $L_{1}$ there exists a continuous function $g: S \rightarrow S$ such that $g(S)=L_{1}$ and $g$ is the identity function on $L_{1}$. Theorem 2 says that there exists $h: L_{1} \rightarrow D$, a continuous inverse arc function such that $h\left(L_{1}\right)=D$. Let $f: S \rightarrow D$ be the continuous function from $S$ onto $D$ such that $f=h g$. Let $L$ be any arc in $D$. Since $h$ is an inverse arc map, there exists an are $L_{2} \subset L_{1}$ such that $h\left(L_{2}\right)=L$. The definition of $f$ implies that

$$
f\left(L_{2}\right)=h\left(g\left(L_{2}\right)\right)=h\left(L_{2}\right)=L .
$$

Thus, $f$ is an inverse arc map and the theorem is proved.

Corollary 3. If $D_{1}$ is any dendrite and $D$ is any dendrite with only a finite number of endpoints, then there exists $f: D_{1} \rightarrow D$, a continuous inverse arc function such that $f\left(D_{1}\right)=D$.

Proof. The proof is obtained by using Lemma 4 and Theorem 7.

Let $D$ and $D_{1}$ be dendrites with infinitely many endpoints. The question that is interesting in this case is: "Does there exist $f$ : $D_{1} \rightarrow D$, a continuous inverse arc function such that $f\left(D_{1}\right)=D$ ?" The only portion of this question that is answered in this paper is when $D_{1}$ has countably many endpoints and $D$ has uncountably many endpoints. For this, Corollary 2 reveals the answer to be no. Thus, there are three cases for further study.

3. A dimension related problem. This section gives a partial answer to the dimension raising ability of a continuous inverse arc function whose domain is a dendrite.

THEOREM 8. If $D$ is a dendrite with only a countable number of endpoints, then there does not exist a continuous inverse arc function, $f$, with domain $D$ such that $\operatorname{dim} f(D) \geqq 2$. 
Proof. Let $D$ be a dendrite and suppose that there does exist a continuous inverse arc function, $f$, with domain $D$ and $\operatorname{dim} f(D) \geqq 2$. Pick a point $p \in f(D)$ at which $f(D)$ has dimension larger than one. The space $f(D)$ has a convex metric $\rho$, [2]. Using this convex metric to generate the topology of $f(D)$ and using the definition of dimension, there exists $\varepsilon>0$ such that the boundary, $B$ of the $\varepsilon$ sphere with center at $p$ is uncountable. Again using the metric $\rho$, we can construct an arc $p y$ for each $y \in B$ such that if $y_{1}, y_{2} \in B$, $y_{1} \neq y_{2}$, then $y_{1} \notin p y_{2}$ and $y_{2} \notin p y_{1}$. Let

$$
M=\bigcup_{y \in B} p y
$$

and then note that Theorem 5 says that $f$ cannot exist and a contradiction is reached.

Corollary 4. If $S$ is a Peano continuum that is the continuous inverse arc image of the unit interval $I$, then $\operatorname{dim} S \leqq 1$.

Proof. Since $I$ is a dendrite with only two endpoints, then Theorem 8 applies.

At this time I have not been able to discover whether or not dimension can be raised on a dendrite with uncountably many endpoints by a continuous inverse arc function. However, Theorem 9 does answer this question in the special case when $\operatorname{dim} f(D)=n$ and $f(D)$ can be imbedded in $E_{n}$.

THEOREM 9. If $D$ is a dendrite, $f$ is an inverse arc function with domain $D, \operatorname{dim} f(D)=n$, and $f(D)$ can be imbedded in $E_{n}$, then $n \leqq 1$.

Proof. If we suppose the contrary, then $n \geqq 2$. Thus, [3], $f(D)$ contains a nonempty open subset of $E_{n}$ and in particular contains an $n$-cube, $n \geqq 2$. Because of this, $f(D)$ contains an uncountable collection, $G$, of mutually exclusive arcs. Since $f$ is an inverse arc function, we can pick an $\operatorname{arc} L_{X} \subset f^{-1}(X)$ for each $X \in G$. Thus, $H=\left\{L_{X}: X \in G\right\}$ is an uncountable collection of mutually exclusive arcs contained in $D$ which contradicts Lemma 3.

\section{REFERENCES}

1. R. H. Bing, Higher-dimensional hereditarily indecomposable continua, Trans. Amer. Math. Soc., 71 (1951), 267-273.

2. - Partitioning a set, Bull. Amer. Math. Soc., 55, (1949), 1101-1110.

3. Hurewicz and Wallman, Dimension Theory, Princeton University Press, Third Printing 1952. 
4. R. L. Moore, Foundations of point set theory, Amer. Math. Soc., Colloq., Vol. 13, Amer. Math. Soc., Providence, R.I. (1962).

5. G. T. Whyburn, Analytic Topology, Amer. Math. Soc., Colloq. Publ., Vol. 28, Amer. Math. Soc., Providence, R. I. (1962).

6. Wilder, Topology of Manifolds, Amer. Math. Soc., Colloq. Publ., Vol. 32, Amer. Math. Soc., Providence, R.I. (1962).

Received December 7, 1971 and in revised form May 5, 1972.

OKLAhoma State UNIVERSity 


\section{PACIFIC JOURNAL OF MATHEMATICS}

EDITORS

\author{
H. SAMELSON \\ Stanford University \\ Stanford, California 94305 \\ C. R. HOBBY \\ University of Washington \\ Seattle, Washington 98105
}

\author{
J. DUGUNDJI \\ Department of Mathematics \\ University of Southern California \\ Los Angeles, California 90007 \\ RICHARD ARENS \\ University of California \\ Los Angeles, California 90024
}

\section{ASSOCIATE EDITORS}

E. F. BECKENBACH

B. H. NeUManN

F. WOLF

K. YosHIDA

\section{SUPPORTING INSTITUTIONS}

UNIVERSITY OF BRITISH COLUMBIA

CALIFORNIA INSTITUTE OF TECHNOLOGY

UNIVERSITY OF CALIFORNIA

MONTANA STATE UNIVERSITY

UNIVERSITY OF NEVADA

NEW MEXICO STATE UNIVERSITY

OREGON STATE UNIVERSITY

UNIVERSITY OF OREGON

OSAKA UNIVERSITY
UNIVERSITY OF SOUTHERN CALIFORNIA

STANFORD UNIVERSITY

UNIVERSITY OF TOKYO

UNIVERSITY OF UTAH

WASHINGTON STATE UNIVERSITY UNIVERSITY OF WASHINGTON

AMERICAN MATHEMATICAL SOCIETY NAVAL WEAPONS CENTER 


\section{Pacific Journal of Mathematics}

\section{Vol. 45, No. $1 \quad$ September, 1973}

William George Bade, Complementation problems for the Baire classes .......... 1

Ian Douglas Brown, Representation of finitely generated nilpotent groups ........ 13

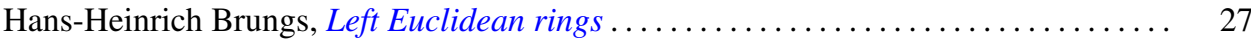

Victor P. Camillo and John Cozzens, A theorem on Noetherian hereditary rings ..... 35

James Cecil Cantrell, Codimension one embeddings of manifolds with locally flat

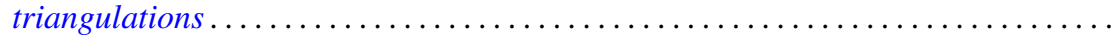

L. Carlitz, Enumeration of up-down permutations by number of rises . . . . . . . . . .

Thomas Ashland Chapman, Surgery and handle straightening in Hilbert cube

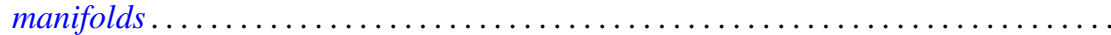

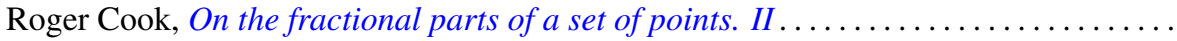

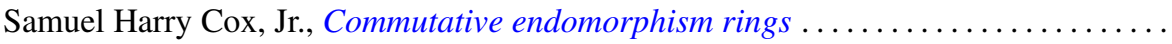

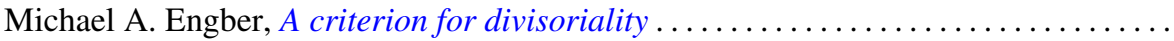

Carl Clifton Faith, When are proper cyclics injective . . . . . . . . . . . . . . 97

David Finkel, Local control and factorization of the focal subgroup . . . . . . . . . 113

Theodore William Gamelin and John Brady Garnett, Bounded approximation by

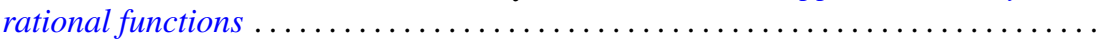

Kazimierz Goebel, On the minimal displacement of points under Lipschitzian

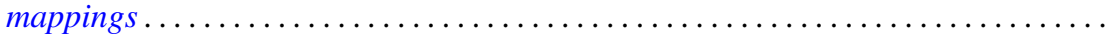

Frederick Paul Greenleaf and Martin Allen Moskowitz, Cyclic vectors for representations associated with positive definite measures: nonseparable

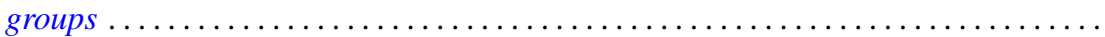

Thomas Guy Hallam and Nelson Onuchic, Asymptotic relations between perturbed linear systems of ordinary differential equations .

David Kent Harrison and Hoyt D. Warner, Infinite primes of fields and completions. .

James Michael Hornell, Divisorial complete intersections . ......

Jan W. Jaworowski, Equivariant extensions of maps ..............

John Jobe, Dendrites, dimension, and the inverse arc function .. .

Gerald William Johnson and David Lee Skoug, Feynman integrals of non-factorable

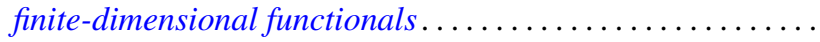

Dong S. Kim, A boundary for the algebras of bounded holomorphic functions ...... 269

Abel Klein, Renormalized products of the generalized free field and its derivatives ... 275

Joseph Michael Lambert, Simultaneous approximation and interpolation in $L_{1}$ and

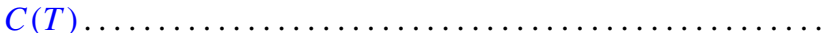

Kelly Denis McKennon, Multipliers of type $(p, p)$ and multipliers of the group

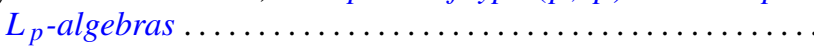

William Charles Nemitz and Thomas Paul Whaley, Varieties of implicative

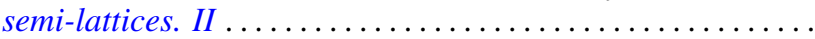

Donald Steven Passman, Some isolated subsets of infinite solvable

Norma Mary Piacun and Li Pi Su, Wallman compactifications on E-completely

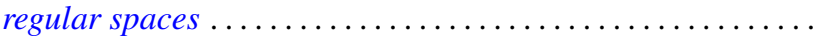

Jack Ray Porter and Charles I. Votaw, $S(\alpha)$ spaces and regular Hausdorff extensions....

Gary Sampson, Two-sided $L_{p}$ estimates of convolution transforms .

Ralph Edwin Showalter, Equations with operators forming a rig
Raymond Earl Smithson, Fixed points in partially ordered sets .

Victor Snaith and John James Ucci, Three remarks on symmetric products and

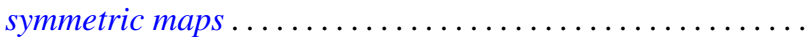

